\title{
Monitoring the Tension and the Temperature in an Overhead Line
}

\author{
M.T. Bedialauneta \\ I. Albizu \\ E. Fernandez A.J. Mazón \\ S. de Arriba \\ Department of Electrical Engineering \\ University of the Basque Country UPV/EHU \\ Bilbao, Spain \\ miren.bedialauneta@ehu.es igor.albizu@ehu.es elvira.fernandezh@ehu.es javier.mazon@ehu.es sdearrib@hotmail.com
}

\begin{abstract}
The paper describes a monitoring system for the evaluation of the low sag behavior of the overhead conductors. The monitoring system measures the conductor tension and temperature. This system includes a methodology for the analysis of the measured data, and obtains the theoretical tension-temperature curve that estimates the conductor's behavior.
\end{abstract}

\section{INTRODUCTION}

There are two types of outdoor testing which are useful for the qualification and acceptation of HTLS conductors: the outdoor laboratory tests and the operating line tests [3]. In an outdoor laboratory test the conductor is at low voltage and the injected current can be controlled whereas in an operating line test the conductor is at the full line voltage and the current through the line depends on the variation of the load. The operating line test is useful in evaluating potential installation difficulties, corrosion and corona issues under outdoor conditions and in testing proposed installation guidelines and maintenance rules whereas the outdoor laboratory test is useful in measuring the sag, the tension and the conductor temperature under sustained high current conditions and naturally varying weather conditions.

Some examples of operating lines tests are shown in [4-7]. In [4] a project where different types of HTLS conductors are installed in several lines is presented. The sag, the tension, the current and the weather magnitudes are measured every 10 minutes. Besides, the splice resistance, the conductor and hardware temperature, the corona, the EMF and the visual appearance are evaluated in periodical site visits. The vibration is also recorded and downloaded during site visits. One of the lines of the project presented in [4] is described in [6]. In this case, gap-type and invar core conductors are monitored. In [5] an ACCR conductor is monitored. The tension and the weather magnitudes are measured every 10 minutes. One of the main drawbacks of the operating line tests is observed in this case. This is the difficulty to obtain high temperature data because of the dependence on the line load. In [7], another example of the monitoring of an ACCR conductor is shown. In this case, the conductor temperature and the weather magnitudes are measured.

This paper describes a monitoring system and methodology for the operating line testing [8] and presents the results obtained in an operating line test. From the results, a methodology for defining the tension-temperature curve that represents the conductor in the operating line test is developed.

\section{OPERATING LINE TEST}

A monitoring system has been installed in an operating line. The monitoring system measures the conductor tension and temperature and it also measures the wind speed to evaluate the conductor load due to wind. The line is a distribution line of $30 \mathrm{kV}$ with an ACSR conductor (147AL1/34-ST1A).

The installed monitoring system (Fig. 1) consists of a sensor for measuring the conductor surface temperature, an ultrasonic anemometer for measuring the speed and the direction of the wind, a load cell for measuring the value of the mechanical tension of the electrical conductor, a temperature sensor for measuring the ambient temperature and a solar radiation sensor that allows measuring the solar radiation.

\section{THEORETICAL BEHAVIOUR}

\section{A. Theoretical model}

The calculated theoretical tension-temperature curve should represent correctly the actual behavior of the conductor. Thus, the behavior of the core and the aluminum as a function of the elastic modulus and CTE should be modeled. The knee-point temperature where the aluminum gets slack should be modeled too. The calculation method used to model the conductor behavior is adapted from sag-tension method developed by some of the authors taking into account the requirements of the HTLS conductors [9-11]. This sag-tension model is simplified for the evaluation of the low sag behavior. Thus, the knee-point temperature is defined and different 
conductor behavior is considered above and below this temperature. Below the knee-point temperature the conductor area, elastic modulus and coefficient of expansion values are taken, and above the core values. The creep deformation is assumed to be constant. As a result, the parameters included in the sag-tension model are the following:

- Span length: $s$

- Conductor weight: $\omega_{\text {con }}$

- Elastic modulus of the conductor and the core: $E_{c o n}$, $E_{\text {core }}$

- Area of the conductor and the core: $A_{\text {con }}, A_{\text {core }}$

- Coefficient of expansion of the conductor and the core: $\alpha_{\text {con }}, \alpha_{\text {core }}$

- Knee-point temperature: $\theta_{\text {knee }}$

The model also needs a tension-temperature reference $T_{o^{-}}$ $\theta_{\mathrm{o}}$. This is obtained from measured values at the beginning of the period of analysis.

The values of the parameters of the operating line are the following:

- $s=100 \mathrm{~m}$

- $\omega_{\text {con }}=0.676 \mathrm{~kg} / \mathrm{m}$

- $E_{\text {con }}=8200 \mathrm{~kg} / \mathrm{mm}^{2}, E_{\text {core }}=19000 \mathrm{~kg} / \mathrm{mm}^{2}$

- $A_{\text {con }}=181.6 \mathrm{~mm}^{2}, A_{\text {core }}=34.3 \mathrm{~mm}^{2}$

- $\alpha_{\text {con }}=17.8 \cdot 10^{-6}{ }^{\circ} \mathrm{C}^{-1}, \alpha_{\text {core }}=11.5 \cdot 10^{-6}{ }^{\circ} \mathrm{C}^{-1}$

- The knee-point temperature $\theta_{\text {knee }}$ is unknown

The tension-temperature reference $T_{o}-\theta_{\mathrm{o}}$ is $382 \mathrm{~kg}-18^{\circ} \mathrm{C}$. It has been obtained from measured values at the beginning of the period of analysis.

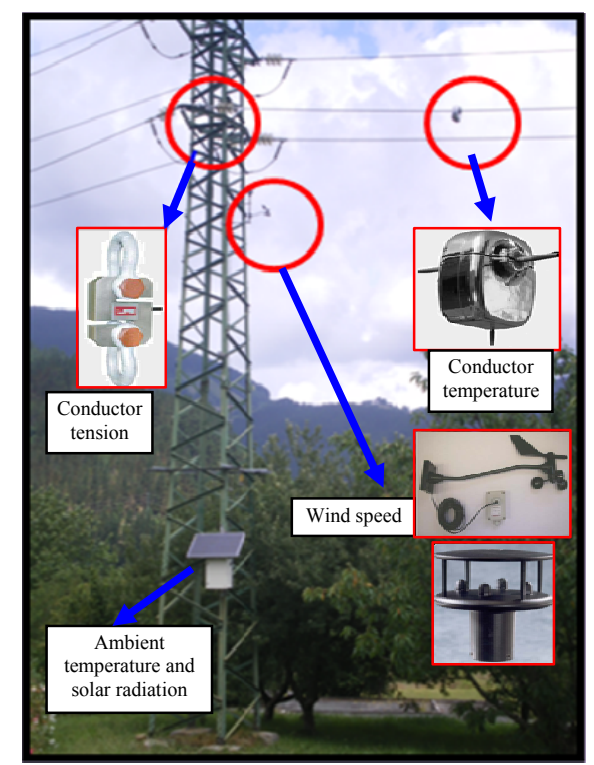

Figure 1. Operating line test
There is an uncertainty in the tension-temperature values given by the model due to the uncertainty in the values of the parameters and the value of the tension-temperature reference.

According to [12], the nominal value for conductor weight corresponds to the minimum acceptable weight and the actual weight typically exceeds the nominal value by $0.2 \%$ to $0.6 \%$. During a conductor's life, the conductor's mass typically increases slightly by tarnishing effects. Also, the weight of a wet conductor can exceed the dry-weight by $1.5 \%$ to $2.5 \%$. The uncertainties yield a typical sag calculation error of $1 \%$ to $2 \%$ for even the simplest single span line [12].

\section{MEASURED BEHAVIOUR}

Figure 2 shows the conductor tension and temperature values measured in a line in operation during a whole day. The direct relation between the tension and the temperature is obvious. The tension value increases when the temperature value decreases and vice versa.

The tension-temperature values that represent the actual behavior of the conductor in operation are obtained processing the acquired measurements. To minimize the effect of the fluctuations of the measured values, the measurements are valid for the analysis of the conductor behavior only if these fluctuations are low. For this purpose, the standard deviation of the measurements is calculated during a defined period of time and if the deviation is below a threshold value the period is valid. If the period is valid the mean values of the measurements are calculated and the results are recorded in the database of the tension-temperature values that represent the behavior of the conductor. Figure 3 shows the standard deviation of the tension measurements (Fig. 2) during a period of time of 30 minutes.
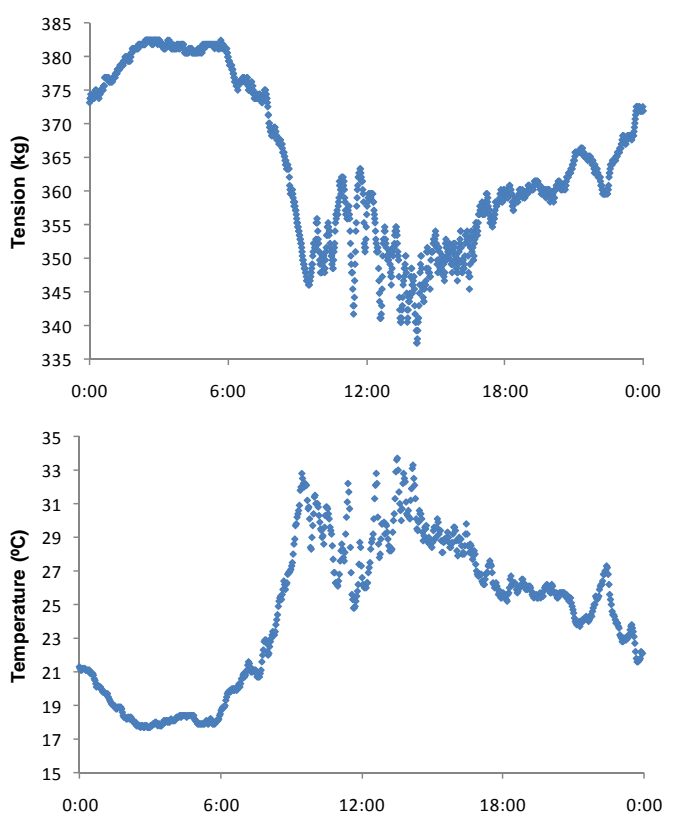

Figure 2. Conductor tension and temperature measured in a line in operation 


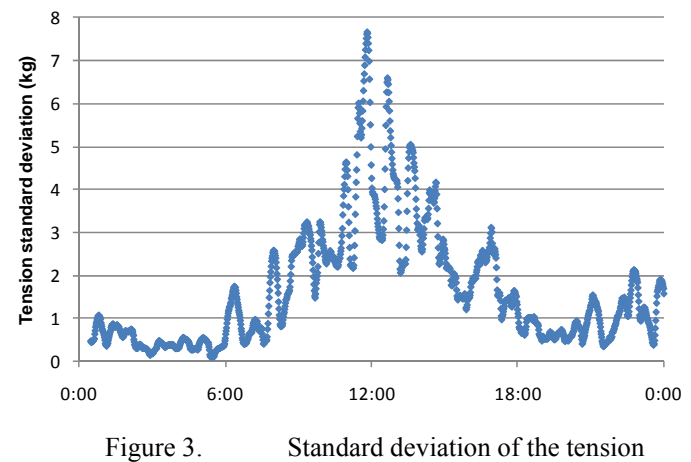

Figure 4 shows the instantaneous measurements obtained for several months. The number of values is 131644 .

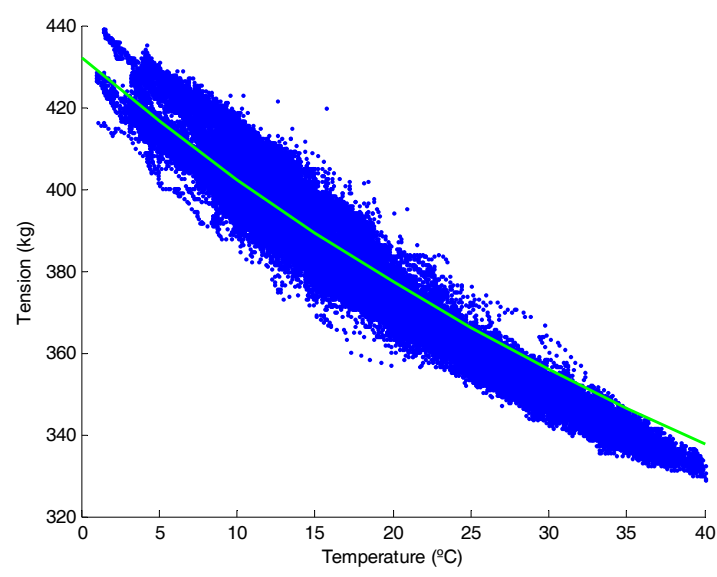

Figure 4. Instantaneous values

These values have filtered using a period of time of 20 minutes and different tension deviation threshold values $(0.5$ $\mathrm{kg}$ and $1 \mathrm{~kg}$ ). The results are shown in Figure 5. It is observed that the higher the tension deviation threshold is, the higher the number of obtained measurements. However, the dispersion of the obtained measurements is also higher. When the threshold decreases, the dispersion is reduced but decreasing the number of measurements and the range of the obtained values. The solid curve corresponds to the theoretical behavior.

Apart from the tension deviation threshold, the value of the period also affects the obtained measurements. The longer is the period it is more difficult that the deviation is below the threshold value. If the obtained values are stable for a longer period they give a higher confidence. Hence, the measurement quality is higher with long periods and low threshold values, but with a lower number of measurements and a smaller range of values.

There are uncertainties in the measured values due to the errors of the measuring systems. In this test, the accuracy of the tension measuring system is $0.6 \mathrm{~kg}$ and the resolution is $0.615 \mathrm{~kg}$. The accuracy of the temperature measuring system is $1{ }^{\circ} \mathrm{C}$ with a resolution of $0.1^{\circ} \mathrm{C}$.
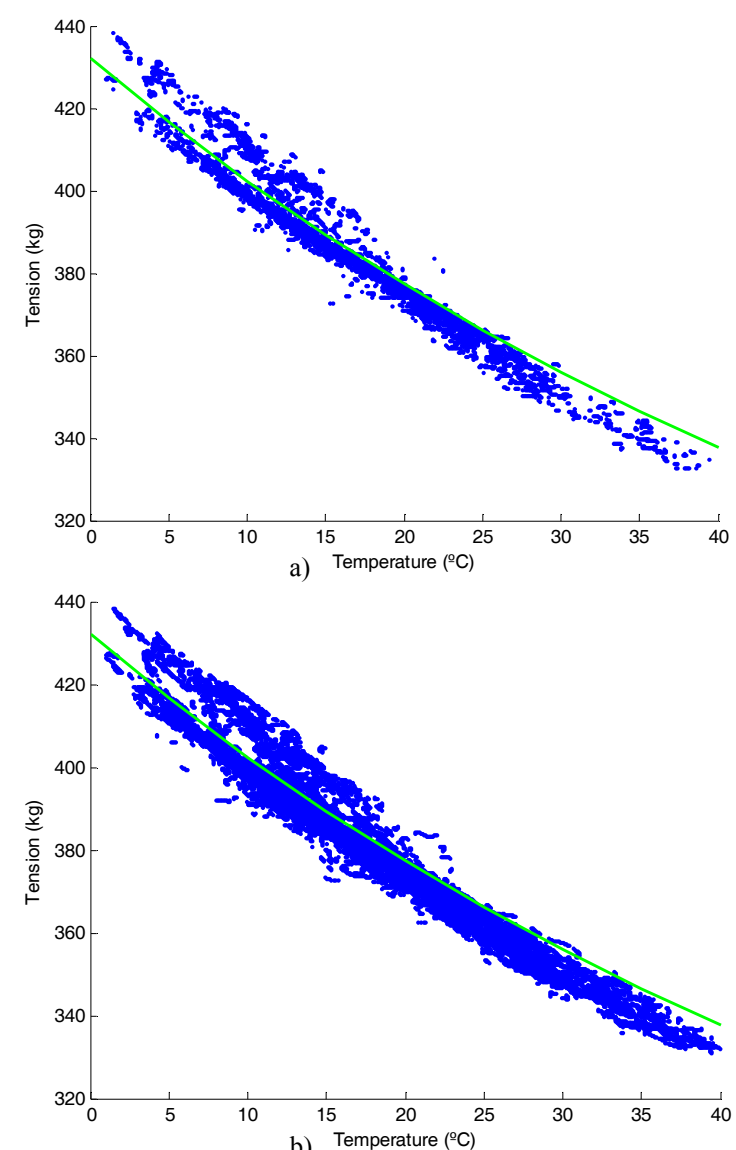

b) Temperature $\left({ }^{\circ} \mathrm{C}\right)$

Figure 5. Theoretical and measured tension-temperature values. Period of time: 20 minutes

a) Deviation threshold: $0.5 \mathrm{~kg}$

b) Deviation threshold: $1 \mathrm{~kg}$

Many of the obtained tension-temperature values correspond to consecutive time periods. For example, if tension-temperature values are obtained from 06:00 to 08:00 with a period of time of 20 minutes and a sampling period of 1 minute, the first value corresponds to the mean value of the measurements carried out between 06:00 and 06:19 and the last value to the mean value of the measurements carried out between 07:41 and 08:00. As a result, there are 101 consecutive values. These values are similar and therefore they could be grouped in a single value. This value is obtained calculating the mean value of the consecutive values. Figure 6 shows the result of grouping the values obtained in Figure 5.a). In this example, when the values are grouped, the number of values is reduced from 28276 in Figure 5.a) to 1778 in Figure 6.

The number of values that contain or represent each grouped value is different. A limit with a minimum of contained values is established in order to eliminate those groups that represent a low number of values. For example, Figure 7 shows the result of establishing a minimum number of 20 represented values. The number of values is reduced from 1778 in Figure 6 to 363 in Figure 7. As a result, the dispersion of the values is also reduced. 


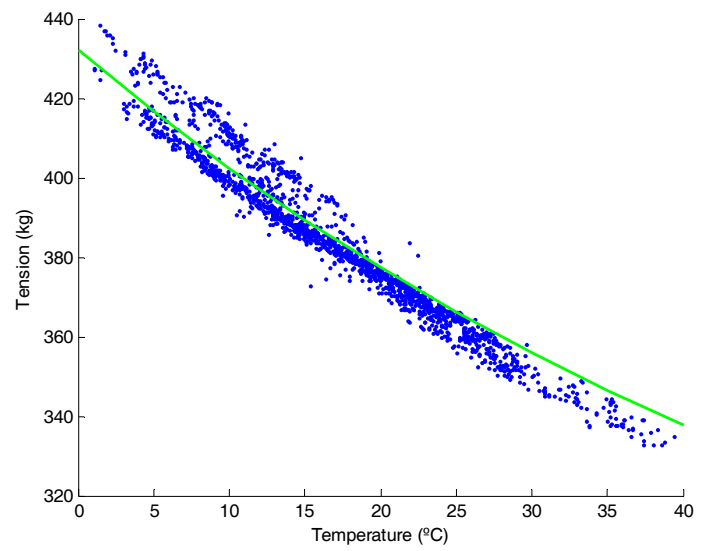

Figure 6. Grouping of values. Period of time 20 minutes and deviation threshold $0.5 \mathrm{~kg}$.

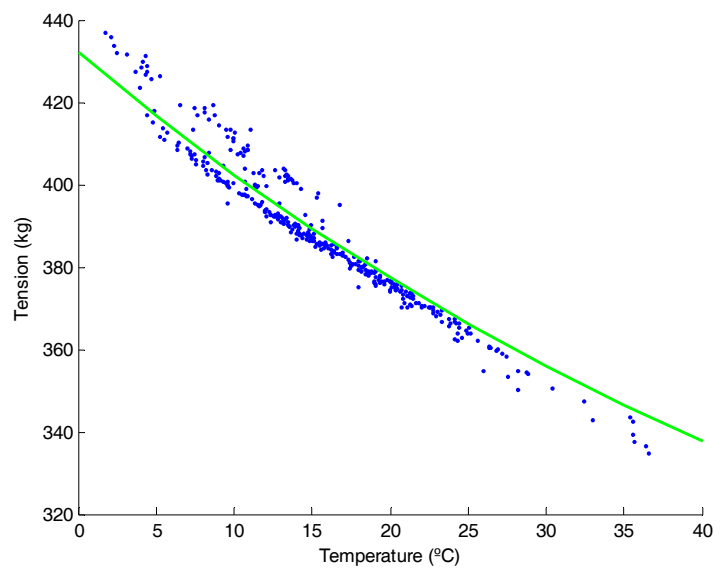

Figure 7. Grouping of values. Minimum represented values: 20

The number of values around $15^{\circ} \mathrm{C}$ are higher than the number of values at lower $\left(5^{\circ} \mathrm{C}\right)$ or higher $\left(35^{\circ} \mathrm{C}\right)$ temperature values. A uniform distribution can be obtained taking only one value for each defined temperature period. For example, a temperature period of $1{ }^{\circ} \mathrm{C}$ can be chosen and the value with highest number of grouped values could be taken. The result is shown in Figure 8.

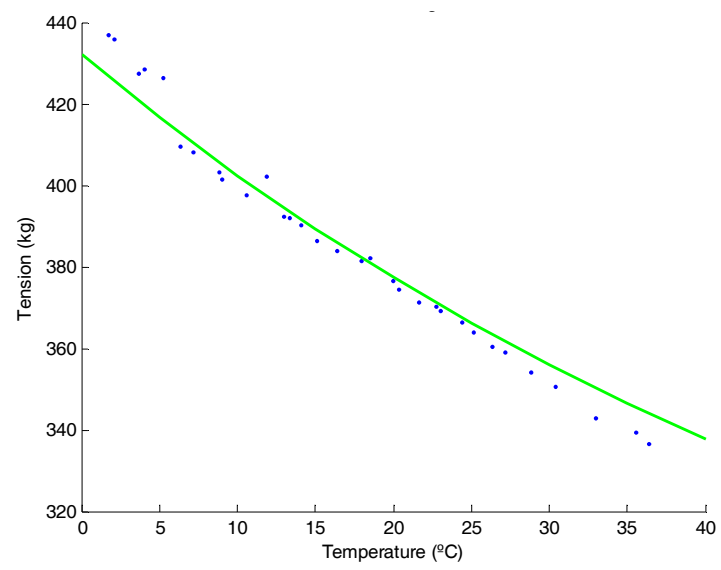

Figure 8. Uniform distribution of values

\section{CONCLUSIONS}

An operating line test for the evaluation of the low sag behavior of the overhead conductors has been carried out. From the results, a methodology for defining the tension-temperature curve that represents the conductor has been developed.

The measured tension-temperature values show a high dispersion around the theoretical behavior. To reduce the dispersion a processing of the measured values is carried out. Firstly, the tension standard deviation is calculated and only the measurement periods with a deviation below a threshold value are taken into account. Secondly, the contiguous values are grouped. From these values, the groups that represent a number of values below a given number are removed. Finally, a uniform distribution is obtained defining uniform temperature periods and taking one value for each period. This representative curve is compared with the curve given by the theoretical model of the conductor.

\section{ACKNOWLEDGMENT}

The authors gratefully acknowledge the support of the Iberdrola utility for the help with the achievement of this project.

\section{REFERENCES}

[1] I. Albizu, A. J. Mazon, and I. Zamora, "Methods for increasing the rating of overhead lines", IEEE PowerTech, St. Petersburg - Russia, 2005.

[2] CIGRE Brochure 244, "Conductors for the Uprating of Overhead Lines", 2004.

[3] CIGRE Brochure 426, "Guide for qualifying high temperature conductors for use on overhead transmission lines", 2010.

[4] B. Clairmont, "High-temperature low-sag transmission conductors", EPRI, 2008.

[5] L. Custer, "DOE-3M demonstration project", IEEE TP \& C Line Design Meeting, Albuquerque-USA, 2006.

[6] A. Goel, "Hydro One Networks", IEEE TP \& C Line Design Meeting, Las Vegas-USA, 2005.

[7] S. Kupke, "Pilot project-High temperature low sag conductors", CIGRE WG B2.42, Stockholm-Sweden, 2010.

[8] I. Albizu, E. Fernandez, A. J. Mazon, M. Bedialauneta and K. Sagastabeitia, "Overhead conductor monitoring system for the evaluation of the low sag behavior", IEEE PowerTech, Trondheim Norway, 2011.

[9] I. Albizu, A. J. Mazon, and I. Zamora, "Flexible strain-tension calculation method for gap-type overhead conductors", IEEE Trans. on Power Del., volume 24, No. 3, pp. 1529-1537, 2009.

[10] I. Albizu, A. J. Mazon, V. Valverde, and G. Buigues, "Aspects to take into account in the application of mechanical calculation to high temperature low sag conductors", IET Gener. Transm. Distrib., volume 4, No. 5, pp. 631-640, 2010.

[11] I. Albizu, A. J. Mazon, and E. Fernandez, "A method for the sagtension calculation in electrical overhead lines", International Review of Electrical Engineering, volume 6, No. 3, pp. 1380-1389, 2011.

[12] CIGRE Brochure 324, "Sag-tension calculation methods for overhead lines", 2007. 